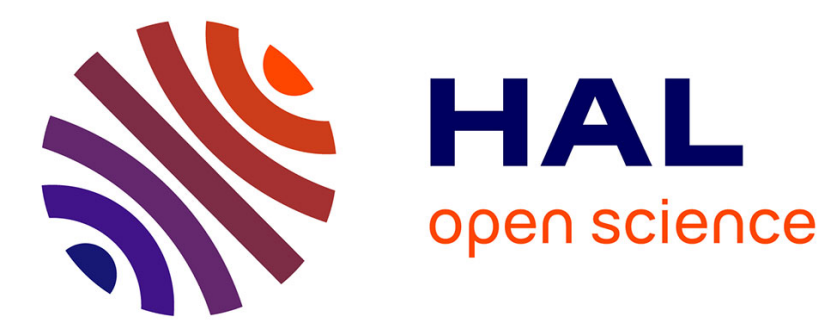

\title{
Application of Interval Observers to Estimation and Control of Air-Fuel Ratio in a Direct Injection Engine
}

Denis Efimov, Shifang Li, Yiran Hu, Steven Muldoon, Hossein Javaherian, Vladimir Nikiforov

\section{- To cite this version:}

Denis Efimov, Shifang Li, Yiran Hu, Steven Muldoon, Hossein Javaherian, et al.. Application of Interval Observers to Estimation and Control of Air-Fuel Ratio in a Direct Injection Engine. Proc. ACC'15, Jul 2015, Chicago, United States. hal-01140347

\section{HAL Id: hal-01140347 \\ https://hal.inria.fr/hal-01140347}

Submitted on 8 Apr 2015

HAL is a multi-disciplinary open access archive for the deposit and dissemination of scientific research documents, whether they are published or not. The documents may come from teaching and research institutions in France or abroad, or from public or private research centers.
L'archive ouverte pluridisciplinaire HAL, est destinée au dépôt et à la diffusion de documents scientifiques de niveau recherche, publiés ou non, émanant des établissements d'enseignement et de recherche français ou étrangers, des laboratoires publics ou privés. 


\title{
Application of Interval Observers to Estimation and Control of Air-Fuel Ratio in a Direct Injection Engine
}

\author{
Denis Efimov, Shifang Li, Yiran Hu, Steven Muldoon, Hossein Javaherian, Vladimir O. Nikiforov
}

\begin{abstract}
The problem of air-to-fuel ratio regulation for a direct injection engine is addressed. A LPV model of the engine is used, for which an interval observer is designed. The interval observer is applied for the model validation and control synthesis. The results of design are confirmed by implementation.
\end{abstract}

\section{INTRODUCTION}

One of the main constraints for engine development and certification is constituted by the requirements on vehicle emission. The fuel-to-air ratio (FAR) regulation is a related engine control problems. FAR control performance can strongly impact key vehicle attributes such as emissions, fuel economy, and drivability. The FAR is regulated at stoichiometric value such that the exhaust gas can be efficiently converted by a three-way-catalyst converter. Thus in the most operating modes of the engine, the primary objective of the on-board FAR control system is to maintain the fuel injection in stoichiometric proportion to the ingested air flow. Variations in the air flow are affected by the driver, they serve as an exogenous disturbance to the FAR regulation system. In some direct injection engines, the fuel is injected via a common rail line directly into the combustion chamber of each cylinder, which leads to an increased fuel efficiency and improved emission level [1].

Due to its importance, the problem of FAR regulation for spark ignition engines has attracted significant attention during the last few decades [2]. Adaptive control theory [3], [4], [5], [6], [7], robust and $H_{\infty}$ control approaches [8], [9], gain scheduling [10], fuzzy control systems theory [11], observer based controllers [12], neural network techniques [13], [14], supervisory controls [15], [16], [17], model predictive controllers [18] and learning methods [19], [20] are successfully tested in this particular application. However, the

Efimov D. and Nikiforov V.O. are with Department of Control Systems and Informatics, ITMO University, 49 Kronverkskiy av., 197101 Saint Petersburg, Russia.

Li S., Hu Y., Muldoon S. and Javaherian H. are with the Propulsion Systems Research Lab, General Motors Global R\&D, 30500 Mound Rd, Warren, MI 48090, US.

Efimov D. is with Non-A team @ Inria, Parc Scientifique de la Haute Borne, 40 av. Halley, 59650 Villeneuve d'Ascq, France and CRIStAL (UMRCNRS 9189), Ecole Centrale de Lille, BP 48, Cité Scientifique, 59651 Villeneuve-d'Ascq, France.

This work was supported in part by the Government of Russian Federation (Grant 074-U01) and the Ministry of Education and Science of Russian Federation (Project 14.Z50.31.0031). complexity of the problem (taking into account the variety of engines and their operating conditions) and growing demands on FAR regulation quality in the presence of a significant modeling uncertainty require new solutions. Additional important constraints for implementation are a small number of tuning parameters and clear design guidelines.

In order to deal with the FAR dynamics uncertainty/complexity, an appealing approach is to represent its model in a Linear Parameter-Varying (LPV) form:

$$
\begin{aligned}
& \dot{x}=A(\theta(t)) x+B(\theta(t)) u, \\
& y=C(\theta(t)) x,
\end{aligned}
$$

where $x \in \mathbb{R}^{n}, u \in \mathbb{R}^{m}, y \in \mathbb{R}^{p}$ are the system state, input and output respectively, $\theta \in \mathbb{R}^{q}$ is the vector of scheduling parameters $\left(\theta: \mathbb{R}_{+} \rightarrow \Theta\right.$ where $\Theta \subset \mathbb{R}^{q}$ is a given set, the instant value $\theta(t)$ can be available for measurements or not), $A, B$ and $C$ are matrix functions of corresponding dimensions. The main advantage is that a partial linearity of LPV models allows plenty of methods developed for linear systems to be applied [21].

If the value of $\theta(t)$ is not measured (it means the presence of uncertainties, unknown parameters or/and disturbances), then design of a control law or an observer for (1) is complicated [21]. Indeed, if $\theta(t)$ is available for measurements, then the following observer can be synthesized:

$$
\dot{\hat{x}}=A(\theta(t)) \hat{x}+B(\theta(t)) u+L(\theta(t))[y-C(\theta(t)) \hat{x}],
$$

where $\hat{x} \in \mathbb{R}^{n}$ is the estimate of $x$ and $L$ is the observer gain [21]. This scheme is an extension of Luenberger approach for LPV systems, $\hat{x}(t)$ should converge to $x(t)$ asymptotically or in a finite time. On the other hand, if $\theta(t)$ is not available for measurements, then such an observer is not realizable since the instant values of the matrix functions $A, B$ and $C$ are unknown. Therefore, in such a case another estimation problem can be posed: an observer has to be designed, which is robust with respect to $\theta(t) \in \Theta$, then $\hat{x}(t)$ may converge not to the exact value of $x(t)$, but to some vicinity of $x(t)$, with the size of the vicinity proportional to the size of $\Theta$.

The interval observers provide a solution in this case. An interval observer is a dynamical system that, using inputoutput information and the bounds of the model uncertainties, evaluates the set of admissible values (interval) for the state 
$x(t)$ at each instant of time. In other words, a system

$$
\begin{gathered}
\dot{z}=g(z, y, u), \\
\underline{x}=\underline{h}(z, y, u), \quad \bar{x}=\bar{h}(z, y, u),
\end{gathered}
$$

where $g, \underline{h}, \bar{h}$ are some known functions (dependent on $\Theta$ ) and $z \in \mathbb{R}^{k}$ with $k \geq n$, is called an interval observer if the relations

$$
\underline{x}(t) \leq x(t) \leq \bar{x}(t)
$$

are satisfied for all $t>0$ provided that $\underline{x}(0) \leq x(0) \leq \bar{x}(0)$ (in this definition the relation $\underline{x}(t) \leq x(t)$ is understood elementwise, i.e. $\underline{x}_{i}(t) \leq x_{i}(t)$ for all $\left.1 \leq i \leq n\right)$. The variables $\underline{x}(t)$ and $\bar{x}(t)$ are called lower and upper bounds of $x(t)$ respectively. The value $\bar{x}(t)-\underline{x}(t)$ is the estimated interval width, which is proportional to the size of the system uncertainty, i.e. the set $\Theta$ dimensions. Such an observer may generate an estimate on the state value $x(t)$ (the mean value of the interval $\hat{x}(t)=0.5[\bar{x}(t)+\underline{x}(t)]$, for example) and also it evaluates its own error of estimation since it is guaranteed that $x(t) \in[\underline{x}(t), \bar{x}(t)]$ for all $t \geq 0$. Thus an interval observer gives more information than a conventional pointwise observer. The interval observers have been proposed recently in the seminal work [22], next many extensions have been proposed for continuous-time systems [23], [24], [25] and discrete-time systems [26], [27]. As we can conclude from these references, the interval observers constitute a new and actively developing area of research.

In this work the interval observer methodology is applied for estimation and control of a LPV model of a direct injection engine. The model of FAR dynamics is presented in Section 2. An interval observer is given in Section 3. In Section 4 it is explained how using interval observers it is possible to evaluate the set $\Theta$. A control algorithm is designed based on the obtained interval observer in Section 5. The performance of the synthesized control is evaluated by simulations and implementation experiments.

\section{LPV MODEL OF FAR DYNAMICS}

A simplified LPV mean-valued model for FAR dynamics can be selected for a direct injection engine in the following form [2], [10]:

$$
\begin{gathered}
\tau_{\lambda}(t) \dot{\lambda}(t)+\lambda(t)=\lambda_{E M}(t), \\
\lambda_{E M}(t)=\frac{1}{A F_{\text {stoich }}} \frac{\dot{m}_{a c}(t)}{\dot{m}_{f i}(t)}, \dot{m}_{a c}(t)=g(t) \eta(t), \\
\dot{m}_{f i}(t)=\frac{N(t)}{30} m_{f i}(t),
\end{gathered}
$$

where $\lambda(t)$ is the air-to-fuel ratio estimate available for direct measurements after catalytic converter and $\lambda_{E M}(t)$ is the regulated variable, which represents the FAR in the cylinders (in this case an uncertain time-varying coefficient $\tau_{\lambda}(t)$ corresponds to parameters of the exhaust manifold and lambda-sensor); $\dot{m}_{a c}(t)$ is the intake air flow in the cylinders, which is proportional to time-varying signals $g(t)$ (uncertain) and $\eta(t)$ (it is a function of measured variables, like the engine rotation velocity $N(t)$, pressures and temperatures in intake and exhaust manifolds, positions of cam phasers, etc.); $A F_{\text {stoch }}=14.681$ is a known constant; $\dot{m}_{f i}(t)$ is the injected fuel flow and $m_{f i}(t)$ is the mass of injected fuel, which is the control input in the system. In fact, there is also a delay in the measurements of $\lambda(t)$, which corresponds to the air transportation in the exhaust manifold, and which has been skipped in this model since for its compensation a separately developed predictor is used (its choice is not critical and it is not presented here for brevity of presentation, the predictor is based on the model (2) with average values of uncertain parameters).

Recall, in (2) the signals $\lambda, m_{f i}, N$ and $\eta$ are measured, the parameters $\tau_{\lambda}$ and $g$ are time-varying (their nominal or mean values $\tau_{\lambda}^{*}, g^{*}$ are also given). The variable $\tau_{\lambda}$ represents the exhaust manifold and lambda-sensor dynamics uncertainty (it may also be used for the exhaust manifold delay modeling), in this case $\tau_{\lambda}^{*}$ corresponds to some identified parameter of the sensor. The variable $g$ is introduced in order to take into account the approximation and modeling errors appeared in $\eta(t)$, thus $g^{*}=1$.

It is easy to see, that for $x(t)=\lambda(t), u(t)=m_{f i}^{-1}(t)$ and $\theta(t)=\left[\begin{array}{lll}\tau_{\lambda}(t) & g(t) & N(t)\end{array}\right]^{T}$ the system (2) can be rewritten as a first order LPV model (1), in this case the vector of scheduling parameters $\theta(t)$ is not available for measurements (only $N(t)$ is measured). It is required to design $m_{f i}$ providing boundedness of the regulation error $\left|\lambda_{E M}(t)-1\right| \leq \Delta$ for some $\Delta>0$ and all $t \geq 0$.

In addition, a database is used with all necessary measured signals for a Chevrolet Equinox with a $2.4 \mathrm{~L}$ direct injection engine, all simulation below are performed based on this database.

\section{INTERVAL OBSERVER FOR FAR VARIABLE IN Chevrolet EQuinox}

For the interval observer design we will assume that the admissible intervals for the values of uncertain time-varying parameters $\tau_{\lambda}$ and $g$ are given:

$$
\underline{\tau}_{\lambda} \leq \tau_{\lambda}(t) \leq \bar{\tau}_{\lambda}, \underline{g} \leq g(t) \leq \bar{g} \quad \forall t \geq 0,
$$

where $\underline{\tau}_{\lambda}, \bar{\tau}_{\lambda}, \underline{g}$ and $\bar{g}$ are known positive constants.

Due to the system structure it is possible to design two interval estimators for the variable $\lambda_{E M}(t)$, one is dependent only on the measured output information, another is based on the input signals only (and intervals of uncertainty defined in (3)).

For the interval observer from the "output", we calculate derivative $\hat{\dot{\lambda}}(t)$ of $\lambda(t)$ using differentiator from [28] or from [29]:

$$
\begin{gathered}
\dot{z}_{1}(t)=-\alpha \sqrt{\left|z_{1}(t)-\lambda(t)\right|} \operatorname{sign}\left[z_{1}(t)-\lambda(t)\right]+z_{2}(t), \\
\dot{z}_{2}(t)=-\beta \operatorname{sign}\left[z_{1}(t)-\lambda(t)\right]-\chi \operatorname{sign}\left[z_{2}(t)\right]-z_{2}(t), \\
\hat{\dot{\lambda}}(t)=z_{2}(t),
\end{gathered}
$$

where $z_{1}, z_{2} \in \mathbb{R}$ are the states of the differentiator, $\beta>$ $\alpha>\chi>0$ are the parameters to be tuned. Then the 
admissible interval $\left[\underline{\lambda}_{E M}^{o}(t), \bar{\lambda}_{E M}^{o}(t)\right]$ of the value $\lambda_{E M}(t)$ can be calculated as follows:

$$
\begin{aligned}
& \underline{\lambda}_{E M}^{o}(t)=\lambda(t)+\pi\left[\hat{\dot{\lambda}}(t), \bar{\tau}_{\lambda}, \underline{\tau}_{\lambda}\right], \\
& \bar{\lambda}_{E M}^{o}(t)=\lambda(t)+\pi\left[\hat{\dot{\lambda}}(t), \underline{\tau}_{\lambda}, \bar{\tau}_{\lambda}\right],
\end{aligned}
$$

where

$$
\pi(\lambda, \underline{a}, \bar{a})= \begin{cases}\bar{a} \lambda, & \text { if } \lambda \geq 0, \\ \underline{a} \lambda, & \text { if } \lambda<0 .\end{cases}
$$

For the interval observer from the "input", we calculate the interval of admissible values $\left[\underline{\dot{m}}_{a c}(t), \overline{\dot{m}}_{a c}(t)\right]$ for the variable $\dot{m}_{a c}(t)$ :

$$
\underline{\dot{m}}_{a c}(t)=\underline{g} \eta(t), \overline{\dot{m}}_{a c}(t)=\bar{g} \eta(t),
$$

where we used the fact that $\eta$ takes only positive values. Next, the FAR channel dynamics can be rewritten as follows:

$$
\lambda_{E M}(t)=\frac{1}{d(t) \dot{m}_{f i}(t)}, d(t)=\frac{A F_{\text {stoich }}}{\dot{m}_{a c}(t)} .
$$

Thus (5) is a static system with measured input $\dot{m}_{f i}(t)$ and known interval bounds for the parameter $d(t)$ :

$$
\underline{d}(t)=\frac{A F_{\text {stoich }}}{\dot{m}_{a c}(t)}, \bar{d}(t)=\frac{A F_{\text {stoich }}}{\underline{\dot{m}}_{a c}(t)} .
$$

Then the following interval estimates can be computed $\left(\dot{m}_{f i}(t)\right.$ takes also only positive values):

$$
\begin{gathered}
\lambda_{E M}(t) \in\left[\underline{\lambda}_{E M}(t), \bar{\lambda}_{E M}(t)\right], \\
\underline{\lambda}_{E M}(t)=\max \left\{\underline{\lambda}_{E M}^{o}(t), \underline{\lambda}_{E M}^{i}(t)\right\}, \\
\bar{\lambda}_{E M}(t)=\min \left\{\bar{\lambda}_{E M}^{o}(t), \bar{\lambda}_{E M}^{i}(t)\right\}, \\
\underline{\lambda}_{E M}^{i}(t)=\left[\bar{d}(t) \dot{m}_{f i}(t)\right]^{-1}, \bar{\lambda}_{E M}^{i}(t)=\left[\underline{d}^{i}(t) \dot{m}_{f i}(t)\right]^{-1} .
\end{gathered}
$$

Therefore, $\left[\underline{\lambda}_{E M}^{o}(t), \bar{\lambda}_{E M}^{o}(t)\right]$ is the interval estimate for $\lambda_{E M}(t)$ obtained from the "output", and $\left[\underline{\lambda}_{E M}^{i}(t), \bar{\lambda}_{E M}^{i}(t)\right]$ is the interval estimate derived from the "input". These interval estimates depend on known bounding variables for unknown time-varying parameters $\tau_{\lambda}, g$ and measured signals. The precision of the obtained interval estimates is proportional to the accuracy of differentiation and the initial uncertainty imposed on the system (parameters $\underline{\tau}_{\lambda}, \bar{\tau}_{\lambda}, \underline{g}$ and $\bar{g})$.

The results of simulation are given in Fig. 1, the timevarying parameters $\tau_{\lambda}(t)$ and $g(t)$ are modeled as stochastic signals with uniform distributions in the selected limits $\left[\underline{\tau}_{\lambda}, \bar{\tau}_{\lambda}\right]$ and $[\underline{g}, \bar{g}]$. As we can see from this simulation, the estimates $\underline{\underline{\lambda}}_{E M}(t), \bar{\lambda}_{E M}(t)$ (the blue and magenta lines) correctly envelop $\lambda_{E M}(t)$ (the cyan line). The time axes is represented in events (in all figures in the paper).

\section{VALIDATION OF MODELS USING INTERVAL OBSERVERS}

Previously we have assumed that for all time-varying parameters an interval of admissible values is given:

$$
\tau_{\lambda} \in\left[\underline{\tau}_{\lambda}, \bar{\tau}_{\lambda}\right], g \in[\underline{g}, \bar{g}],
$$

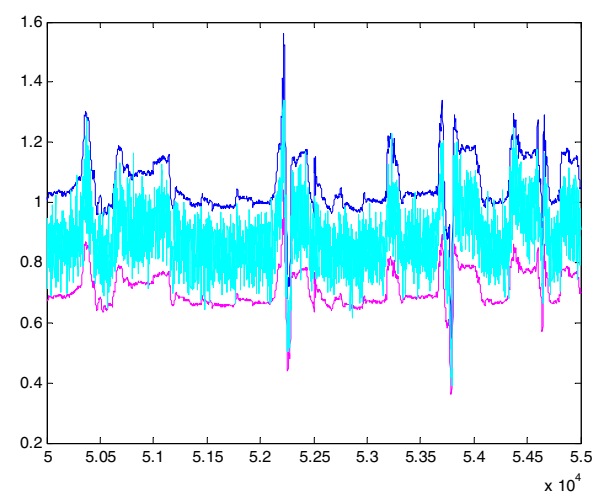

Figure 1. The results of simulation of the interval observer (6)

where $0<\underline{\tau}_{\lambda} \leq \bar{\tau}_{\lambda}, 0<g \leq \bar{g}$ are some constants, which define the admissible deviations of the model parameter from the nominal values. The variables $\tau_{\lambda}, g$ are not measured (they were introduced in order to model approximation errors or uncertain processes), and as it has been noticed, accuracy of the interval estimation is proportional to the model uncertainty, which is represented by the intervals $\left[\underline{\tau}_{\lambda}, \bar{\tau}_{\lambda}\right]$ and $[\underline{g}, \bar{g}]$. Therefore, a good identification of the values $\underline{\tau}_{\lambda}, \bar{\tau}_{\lambda}$, $\bar{g}, \bar{g}$ is very important for application of interval observers. In this section we will show how the values of constants $\underline{\tau}_{\lambda}, \bar{\tau}_{\lambda}$, $\underline{g}, \bar{g}$ can be calculated off-line using datasets of measurements (on which the model was approximated).

The idea is to calculate the interval estimates $\underline{\lambda}(t), \bar{\lambda}(t)$ of the measured variable $\lambda(t)$ using the output of the interval observer (6), then we may adjust $\underline{\tau}_{\lambda}, \bar{\tau}_{\lambda}, \underline{g}, \bar{g}$ such that $\lambda(t) \in$ $[\underline{\lambda}(t), \bar{\lambda}(t)]$ for all $t \geq 0$ (for all recorded samples used for the model approximation and validation), which will be an explicit indication that intervals for $\tau_{\lambda}, g$ have been selected properly.

Using the values of $\underline{\lambda}_{E M}(t), \bar{\lambda}_{E M}(t)$ (which are dependent on $\underline{\tau}_{\lambda}, \bar{\tau}_{\lambda}, g, \bar{g}$ ) we may calculate the interval estimates for the measured output $\lambda$ applying the following interval framer:

$$
\dot{\bar{\lambda}}(t)=\frac{\bar{\lambda}_{E M}(t)}{\underline{\tau}_{\lambda}}-\frac{\bar{\lambda}(t)}{\bar{\tau}_{\lambda}}, \underline{\dot{\lambda}}(t)=\frac{\underline{\lambda}_{E M}(t)}{\bar{\tau}_{\lambda}}-\frac{\underline{\lambda}(t)}{\underline{\tau}_{\lambda}} .
$$

The values $\underline{\tau}_{\lambda}, \bar{\tau}_{\lambda}, g, \bar{g}$ can be tuned using the trial and error method. Initially, the intervals $\left[\underline{\tau}_{\lambda}, \bar{\tau}_{\lambda}\right]$ and $[g, \bar{g}]$ are selected big enough guaranteeing that $\lambda(t) \in[\underline{\lambda}(t), \bar{\lambda}(t)]$ for the given dataset. Next, the width of intervals $\left[\underline{\tau}_{\lambda}, \bar{\tau}_{\lambda}\right]$ and $[\underline{g}, \bar{g}]$ is iteratively decreased while the inclusion $\lambda(t) \in[\underline{\lambda}(\bar{t}), \bar{\lambda}(t)]$ is preserved. For example, in Fig. 2 the results obtained on the dataset for the case of $5 \%$ deviations from the nominal values are shown $\left(\underline{\tau}_{\lambda}=0.95 \tau_{\lambda}^{*}, \bar{\tau}_{\lambda}=1.05 \tau_{\lambda}^{*}\right.$, $\underline{g}=0.95$ and $\bar{g}=1.05$ ). The time-varying parameters $\bar{\tau}_{\lambda}(t)$ and $g(t)$ are modeled as stochastic signals (uniform distributions) with the values in $\left[\underline{\tau}_{\lambda}, \bar{\tau}_{\lambda}\right]$ and $[g, \bar{g}]$. As we can conclude the interval estimates $\underline{\lambda}(t), \bar{\lambda}(t)$ (the red lines) nicely envelop the signal $\lambda(t)$ (the magenta line), the estimate $\hat{\lambda}_{E M}(t)=0.5\left[\bar{\lambda}_{E M}(t)+\underline{\lambda}_{E M}(t)\right]$ is also shown (the blue line). This technique gives us an evaluation of the parameter 


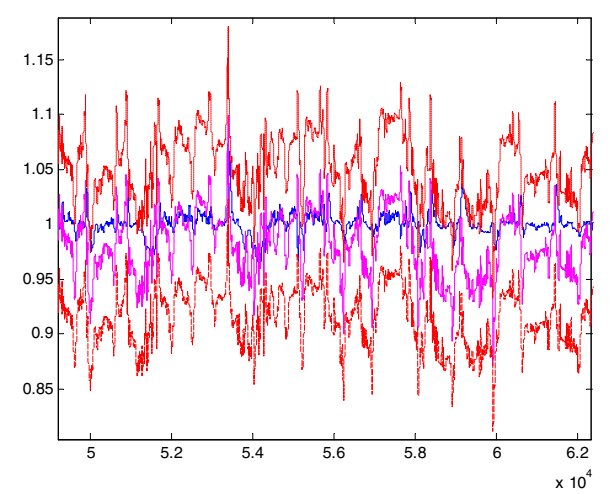

Figure 2. The signals $\lambda$ (magenta), $\hat{\lambda}_{E M}$ (blue) and $\bar{\lambda}, \underline{\lambda}$ (red)

uncertainty acceptable for the model representation.

\section{FAR CONTROL}

By design of the control $m_{f i}$ it is required to ensure the relation $\lambda_{E M}(t)=1$ with the maximal admissible deviation from this value $25 \%$, i.e. $\Delta=0.25$.

\section{A. Control design}

As an auxiliary control strategy let us calculate the values of $\dot{m}_{f i}(t)$ that ensures realization of the control goal $\lambda_{E M}(t)=1$. From (5), we derive the following constraints to guarantee:

$$
\underline{d}(t) \dot{m}_{f i}(t)=1, \bar{d}(t) \dot{m}_{f i}(t)=1,
$$

then $\underline{\lambda}_{E M}^{i}(t)=\bar{\lambda}_{E M}^{i}(t)=1$, and since $\underline{\lambda}_{E M}^{i}(t) \leq$ $\lambda_{E M}(t) \leq \bar{\lambda}_{E M}^{i}(t)$ for all $t \geq 0$ then the control goal has to be achieved. The common control value that minimizes the error for both equations can be found by the formula (a least square solution):

$$
\dot{m}_{f i}^{0}(t)=\frac{\underline{d}(t)+\bar{d}(t)}{2 \underline{d}(t) \bar{d}(t)} .
$$

The control (7) can be used in the "normal" situation, when $\underline{\lambda}_{E M}(t), \bar{\lambda}_{E M}(t)$ do not exceed the admissible $25 \%$ deviations from 1 . If it is not the case, simple correction controls can be used:

$$
\overline{\dot{m}}_{f i}(t)=\bar{\lambda}_{E M}^{*} / \underline{d}(t), \underline{\dot{m}}_{f i}(t)=\underline{\lambda}_{E M}^{*} / \bar{d}(t),
$$

where $\bar{\lambda}_{E M}^{*}, \underline{\lambda}_{E M}^{*} \in[1-\Delta, 1+\Delta]$ are design parameters. The control $\dot{m}_{f i}(t)$ guarantees that $\bar{\lambda}_{E M}^{i}(t)=\bar{\lambda}_{E M}^{*}$, and it can be used if $\underline{\lambda}_{E M}(t)>1+\Delta$ in order to return the trajectory back in the desired domain. The control $\underline{\dot{m}}_{f i}(t)$ similarly ensures $\underline{\lambda}_{E M}^{i}(t)=\underline{\lambda}_{E M}^{*}$, it can be activated if $\bar{\lambda}_{E M}(t)<1-\Delta$. Finally, combining (7) and (8) we obtain the feedforward control algorithm for FAR channel:

$$
m_{f i}^{f f}(t)=\frac{30}{N(t)} \begin{cases}\underline{\dot{m}}_{f i}(t) & \text { if } \bar{\lambda}_{E M}(t)<1-\Delta, \\ \dot{m}_{f i}(t) & \text { if } \underline{\lambda}_{E M}(t)>1+\Delta, \\ \dot{m}_{f i}^{0}(t) & \text { otherwise. }\end{cases}
$$

Lemma 1. For the model (2) with the control (9), if the relations (3) are satisfied for some known positive constants $\underline{\tau}_{\lambda}, \bar{\tau}_{\lambda}, \underline{g}$ and $\bar{g}$, then

$$
\begin{aligned}
& \lambda_{E M}(t) \in\left[\min \left\{1-\Delta_{g}, \frac{1}{\underline{\lambda}_{E M}^{*}}, \frac{\underline{g}}{\bar{\lambda}_{E M}^{*} \bar{g}}\right\},\right. \\
&\left.\max \left\{1+\Delta_{g}, \frac{\bar{g}}{\underline{\lambda}_{E M}^{*} \underline{g}}, \frac{1}{\bar{\lambda}_{E M}^{*}}\right\}\right]
\end{aligned}
$$

for all $t \geq 0$, where $\Delta_{g}=\frac{\bar{g}-g}{\underline{g}+\overline{\bar{g}}}$.

Proof. Note that

$$
\lambda_{E M}\left(t, \dot{m}_{f i}(t)\right)=\frac{g(t) \eta(t)}{A F_{\text {stoich }}} \frac{1}{\dot{m}_{f i}(t)},
$$

then for the control (7) we obtain

$$
\begin{aligned}
\lambda_{E M}^{0}(t) & =\lambda_{E M}\left(t, \dot{m}_{f i}^{0}(t)\right) \\
& =\frac{g(t) \eta(t)}{A F_{\text {stoich }}} \frac{2 \underline{d}(t) \bar{d}(t)+\bar{d}(t)}{d(t)}=\frac{2 g(t)}{\underline{g}+\bar{g}},
\end{aligned}
$$

and, consequently, $\lambda_{E M}^{0}(t) \in\left[\frac{2 \underline{g}}{\underline{g}+\bar{g}}, \frac{2 \bar{g}}{\underline{g}+\bar{g}}\right]=\left[1-\Delta_{g}, 1+\Delta_{g}\right]$. For the controls (8) we get

$$
\begin{aligned}
& \underline{\lambda}_{E M}^{*}(t)=\lambda_{E M}\left(t, \underline{\dot{m}}_{f i}(t)\right)=\frac{g(t)}{\underline{\lambda}_{E M}^{*} \underline{g}}, \\
& \bar{\lambda}_{E M}^{*}(t)=\lambda_{E M}\left(t, \overline{\dot{m}}_{f i}(t)\right)=\frac{g(t)}{\bar{\lambda}_{E M}^{*} \bar{g}},
\end{aligned}
$$

then $\underline{\lambda}_{E M}^{*}(t) \in\left[\frac{1}{\underline{\lambda}_{E M}^{*}}, \frac{\bar{g}}{\underline{\lambda}_{E M}^{*} g}\right]$ and $\bar{\lambda}_{E M}^{*}(t) \in\left[\frac{\underline{g}}{\lambda_{E M}^{*} \bar{g}}, \frac{1}{\lambda_{E M}^{*}}\right]$. Since the values of $\lambda_{E M}(t)$ with the control (9) are in the set $\left\{\lambda_{E M}^{0}(t), \underline{\lambda}_{E M}^{*}(t), \bar{\lambda}_{E M}^{*}(t)\right\}$, then the required interval estimate follows.

Corollary 1. Under the conditions of Lemma 1, if $\frac{\bar{g}}{\underline{g}} \leq$ $\frac{1+\Delta}{1-\Delta}$, then there exist $\underline{\lambda}_{E M}^{*} \in\left[\frac{\bar{g}}{(1+\Delta) \underline{g}}, \frac{1}{1-\Delta}\right]$ and $\bar{\lambda}_{E M}^{*} \in$ $\left[\frac{1}{1+\Delta}, \frac{g}{(1-\Delta) \bar{g}}\right]$ such that $\lambda_{E M}(t) \in[1-\Delta, 1+\Delta]$ for all $t \geq 0$.

Proof. The claim follows by a direct substitution.

For feedback regulation, an estimate of the regulated variable value is needed, and for this purpose we will use

$$
\hat{\lambda}_{E M}(t)=0.5\left[\bar{\lambda}_{E M}^{o}(t)+\underline{\lambda}_{E M}^{o}(t)\right]=\lambda(t)+\tau_{\lambda}^{*} \hat{\dot{\lambda}}(t) .
$$

Let us stress that the estimates $\underline{\lambda}_{E M}^{i}(t), \bar{\lambda}_{E M}^{i}(t)$, which are dependent on the control signals, were used for calculation of $m_{f i}$. Then the following nonlinear PID, based on the estimates $\bar{\lambda}_{E M}^{o}(t), \underline{\lambda}_{E M}^{o}(t)$, can be used for the feedback:

$$
\begin{aligned}
m_{f i}^{f b}(t)= & k_{1}\lceil e(t)\rfloor^{p_{1}}+k_{2} \int_{0}^{t}\lceil e(s)\rfloor^{p_{2}} d s+k_{3} \hat{\dot{e}}(t) \\
& +k_{4} \operatorname{sign}[e(t)]+k_{5}\lceil e(t)\rfloor^{p_{3}}
\end{aligned}
$$

where $\lceil e(t)\rfloor^{p}=|e(t)|^{p} \operatorname{sign}[e(t)], e(t)=1-\hat{\lambda}_{E M}(t)$ is the regulation error, $\hat{\dot{e}}(t)$ is an estimate of the derivative of $e(t)$ calculated by differentiator (4), the coefficients $k_{i}$, $i=1, \ldots, 5$ and powers $0<p_{1} \leq 1, p_{2} \leq 1,1 \leq p_{3}$ have to be adjusted using the trial and error method. The terms proportional to $k_{1}$ and $k_{5}$ are responsible for proportional 


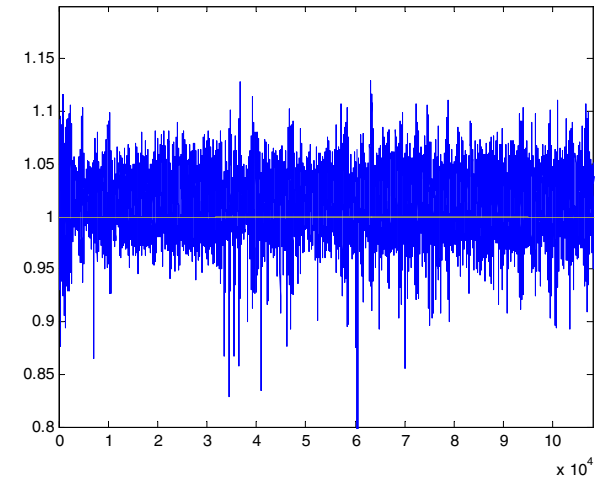

Figure 3. AFR $\lambda$ regulation in simulation

regulation in local and global scale correspondingly, if $p_{1}<1$ and $p_{3}>1$ then a fixed-time rate of convergence can be guaranteed [30] (it is better to use different powers, $p_{1} \leq 1$ for local regulation and $p_{3} \geq 1$ for global setting; in the conventional PID $p_{1}=p_{3}=1$ then $k_{1}=k_{5}$ can be selected). The coefficients $k_{2}$ and $k_{3}$ correspond to integral and derivative actions in PID, the power $p_{2}<1$ may ensure a finite-time rate of static error compensation. The sliding mode term proportional to $k_{4}$ serves for cancellation of matched uncertainties.

The final expression of the control is:

$$
m_{f i}(t)=\operatorname{sat}\left[m_{f i}^{f f}(t)+m_{f i}^{f b}(t)\right],
$$

where $\operatorname{sat}[\cdot]$ is a saturation function, which ensures that the control lies in the admissible bounds.

\section{B. Results of simulation}

The results of simulation are shown in Fig. 3, the timevarying parameters $\tau_{\lambda}(t)$ and $g(t)$ are modeled as stochastic signals in the required limits $( \pm 5 \%$ of deviation, in this case the conditions of Lemma 1 and Corollary 1 are satisfied). In Fig. 3 the variable $\lambda(t)$ (blue line) and its reference value (yellow line) are plotted. As we can conclude, the regulation error is rather small, that confirms the obtained theoretical results in Lemma 1 and Corollary 1. An example of the interval observer (6) estimates is given in Fig. 1. Thus the proposed interval observer based control demonstrated sufficient robustness and accuracy in the simulations.

\section{Results of implementation}

The plots of signals obtained during an US06 cycle (this test cycle includes sufficiently different driving conditions) for Chevrolet Equinox (2.4L direct injection engine) are shown in figures 4-6 (throttle position control has been generated by ECM in this case). As we can conclude from these results, in Fig. 5 the variable $\lambda(t)$ lies in the limits of $25 \%$ deviation from the desired value 1 during the complete cycle, and almost everywhere the more advantageous limits in $10 \%$ of deviation are guaranteed. The estimates of the regulated variable $\lambda_{E M}(t)$ given in Fig. 6 are almost all time

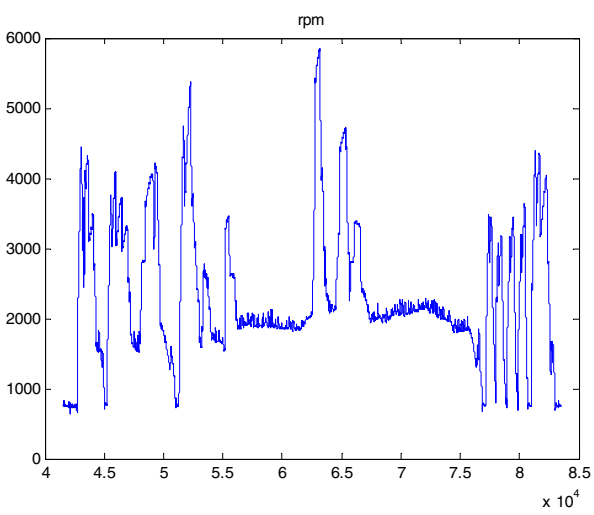

Figure 4. $N(t)$ in implementation

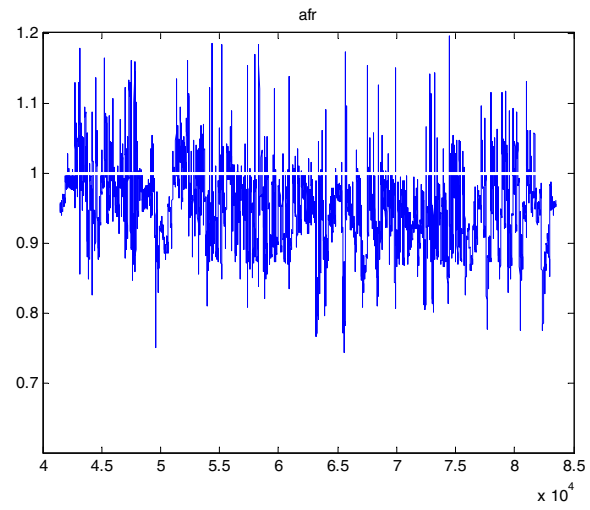

Figure 5. AFR in implementation

in the correct limits (blue line is the estimate $\hat{\lambda}_{E M}(t)$, red and green lines are $\underline{\lambda}_{E M}(t)$ and $\bar{\lambda}_{E M}(t)$ respectively). Thus the developed FAR control algorithm based on interval observer has acceptable performance in the presence of diverse engine operating modes, which can be seen analyzing Fig. 4, where the engine rotation speed $N(t)$ is presented. In addition, it is worth to stress that in the tests only feedforward control has been used, the feedback control has been switched off (i.e. $k_{i}=0$ for all $\left.i=1, \ldots, 5\right)$, the reason for that is to show advantages and workability of the interval observer based scheme.

\section{CONCLUSION}

Interval observers constitute a new method for state estimation of significantly uncertain systems. These observers provide an estimate on the state and compute bounds on estimation error simultaneously, i.e. at each instant of time they evaluate the interval of admissible values of state (that is a counterpart of the Kalman filtering in deterministic setting). Width of the estimated interval is proportional to the current size of uncertainty in the system description. An important area of applications for interval observers deals with LPV systems (especially in the case with unmeasured vector of scheduling parameters). A simple LPV model of spark ignition engine is analyzed in the paper. An interval 


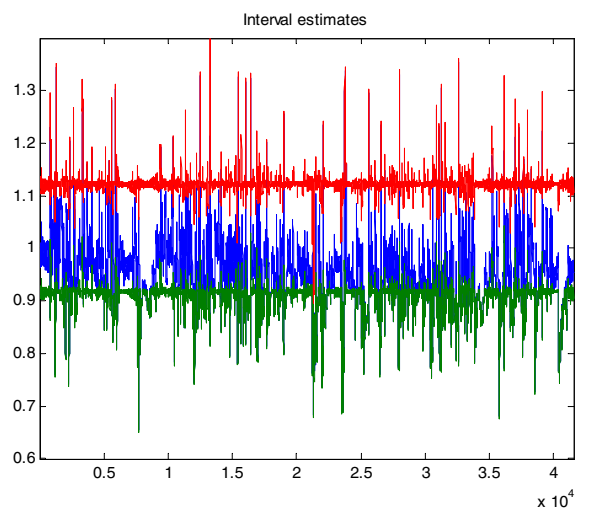

Figure 6. Estimates of $\lambda_{E M}$ in implementation

observer is designed for this model. It is shown how this interval observers can be used for estimation of the set of admissible values for unmeasured elements of the vector of scheduling parameters. Finally, control algorithm is designed based on interval estimates, the analytical performance conditions are also derived. The efficiency of the proposed estimation and control algorithms is confirmed by the results of implementation with US 06 tests for Chevrolet Equinox.

\section{ACKNOWLEDGMENTS}

The financial support for this research project was provided by General Motors Company and is gratefully acknowledged by the lead author and Prof. Nikiforov of University ITMO, Russia.

\section{REFERENCES}

[1] H. Zhao, Advanced Direct Injection Combustion Engine Technologies and Development: Gasoline and Gas Engines. CRC Press, 2010.

[2] J. Cook, I. Kolmanovsky, D. McNamara, E. Nelson, and K. Prasad, "Control, computing and communications: Technologies for the twenty-first century model T," Proc. of the IEEE, vol. 95, no. 2, pp. 334-355, 2007.

[3] B. Ault, V. Jones, J. Powell, and G. Franklin, "Adaptive air-fuel ratio control of a spark ignition engine," SAE Technical Paper, no. 940373, 1994.

[4] E. Franceschi, K. Muske, J. Peyton-Jones, and I. Makki, "An adaptive delay-compensated pid air fuel ratio controller," in SAE Technical Paper 2007-01-1342, 2007.

[5] J. Powell, N. Fekete, and C.-F. Chang, "Observer-based air fuel ratio control," IEEE Control Systems Magazine, vol. 18, no. 5, pp. 72-83, 1998.

[6] R. Turin and H. Geering, "Model-reference adaptive a/f-ratio control in an si engine based on kalman-filtering techniques," in Proc. American Control Conference, vol. 6, 1995, pp. 4082-4090.

[7] Y. Yildiz, A. Annaswamy, D. Yanakiev, and I. Kolmanovsky, "Adaptive air fuel ratio control for internal combustion engines," in Proc. American Control Conference, 2008, pp. 2058-2063.

[8] M. Brandstetter, "Robust air-fuel ratio control for combustion engines," Ph.D. dissertation, Cambridge, UK, 1996.

[9] L. Mianzo, H. Peng, and I. Haskara, "Transient air-fuel ratio $H_{\infty}$ preview control of a drive-by-wire internal combustion engine," in Proc. American Control Conference, vol. 4, 2001, pp. 2867-2871

[10] A. di Gaeta, U. Montanaro, and V. Giglio, "Model-based control of the air fuel ratio for gasoline direct injection engines via advanced cosimulation: An approach to reduce the development cycle of engine control systems," J. Dyn. Sys., Meas., Control, vol. 133, no. 6, p. 061006, 2011.
[11] A. Ghaffari, A. Shamekhi, A. Saki, and E. Kamrani, "Adaptive fuzzy control for air-fuel ratio of automobile spark ignition engine," Proc. World Acad. Science, Engineering, Technology, pp. 1064-1072, 2008.

[12] C.-F. Chang, N. P. Fekete, A. Amstutz, and J. D. Powell, "Air-fuel ratio control in spark-ignition engines using estimation theory," IEEE Trans. Control Systems Technology, vol. 3, no. 1, pp. 22-31, 1995.

[13] T. Huang, D. Liu, H. Javaherian, and N. Sin, "Neural sliding mode control of engine torque," in Proc. 2008 IFAC Triennial World Congress, Seoul, South Korea, 2008.

[14] Y. Zhai and D. Yu, "Rbf based feedforward feedback control for airfuel ratio of si engines," in Proc. IFAC Workshop on Advanced Fuzzy and Neural Control, 2007.

[15] D. Efimov, H. Javaherian, and V. Nikiforov, "Switching control of air-fuel ratio in spark ignition engines," in Proc. American Control Conference, Baltimore, USA, June 30-July 022010.

[16] D. Efimov, V. Nikiforov, and H. Javaherian, "Supervisory control of air-fuel ratio in spark ignition engines," Control Engineering Practice, vol. 30, pp. 27-33, 2014.

[17] S. Kolyubin, D. Efimov, V. Nikiforov, and A. A. Bobtsov, "Twochannel adaptive hybrid control of the air-to-fuel ratio and torque of automobile engines," Automation and Remote Control, vol. 73, no. 11, pp. 1794-1807, 2012

[18] K. Muske and C. Jones, "A model-based si engine air fuel ratio controller," in Proc. American Control Conference, 2006.

[19] D. Rupp and L. Guzzella, "Iterative tuning of internal model controllers with application to air/fuel ratio control," IEEE Trans. Control Systems Technology, vol. 18, no. 1, pp. 177-184, 2010.

[20] D. Efimov, H. Javaherian, and V. Nikiforov, "Iterative learning airfuel ratio control with adaptation in spark ignition engines," in Proc. American Control Conference, 2010, pp. 2063-2068.

[21] J. Mohammadpour and C. Scherer, Eds., Control of Linear Parameter Varying Systems with Applications. Springer, 2012.

[22] J. Gouzé, A. Rapaport, and Z. Hadj-Sadok, "Interval observers for uncertain biological systems," Ecological modeling, vol. 133, pp. 45$56,2000$.

[23] F. Mazenc and O. Bernard, "Interval observers for linear time-invariant systems with disturbances," Automatica, vol. 47, no. 1, pp. 140-147, 2011.

[24] C. Combastel, "Stable interval observers in BBC for linear systems with time-varying input bounds," IEEE Transactions on Automatic Control, vol. 58, no. 2, pp. 481-487, 2013.

[25] T. Raïssi, D. Efimov, and A. Zolghadri, "Interval state estimation for a class of nonlinear systems," IEEE Trans. Automatic Control, vol. 57, no. 1 , pp. 260-265, 2012.

[26] F. Mazenc, T. Dinh, and S. Niculescu, "Interval observers for discretetime systems," in Proc. IEEE CDC 2012, 2012.

[27] D. Efimov, W. Perruquetti, T. Raïssi, and A. Zolghadri, "Interval observers for time-varying discrete-time systems," IEEE Trans. Automatic Control, vol. 58, no. 12, pp. 3218-3224, 2013.

[28] W. Perruquetti, T. Floquet, and E. Moulay, "Finite-time observers: Application to secure communication," IEEE Trans. Automatic Control, vol. 53, no. 1, pp. 356-360, 2008.

[29] D. Efimov and L. Fridman, "A hybrid robust non-homogeneous finitetime differentiator," IEEE Trans. Automatic Control, vol. 56, no. 5, pp. 1213-1219, 2011.

[30] A. Polyakov, "Nonlinear feedback design for fixed-time stabilization of linear control systems," IEEE Trans. Automatic Control, vol. 57, no. 8, pp. 2106-2110, 2012. 